\title{
Narrativa
}

\section{La letteratura ultra-contemporanea italiana in Tunisia: le ragioni di un "incontro impossibile"}

\section{Mario Sei}

\section{(2) OpenEdition}

1 Journals

Edizione digitale

URL: https://journals.openedition.org/narrativa/820

DOI: $10.4000 /$ narrativa.820

ISSN: 2804-1224

Editore

Presses universitaires de Paris Nanterre

\section{Edizione cartacea}

Data di pubblicazione: 1 décembre 2016

Paginazione: $77-87$

ISBN: 978-2-84016-266-7

ISSN: $1166-3243$

Notizia bibliografica digitale

Mario Sei, «La letteratura ultra-contemporanea italiana in Tunisia: le ragioni di un "incontro

impossibile"», Narrativa [Online], 38 | 2016, online dal 01 janvier 2022, consultato il 22 février 2022.

URL: http://journals.openedition.org/narrativa/820 ; DOl: https://doi.org/10.4000/narrativa.820

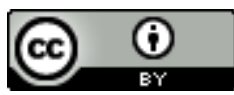

Narrativa est mise à disposition selon les termes de la Licence Creative Commons Attribution 4.0 International. 


\section{La letteratura ultra-contemporanea italiana in Tunisia: le ragioni di un "incontro impossibile"}

n quante zone del mondo il fatto stesso di porsi la domanda sulla diffusione e la ricezione della letteratura italiana ultra-contemporanea sarebbe a dir poco incongruo, se non del tutto assurdo?

Questa domanda, sorta quasi automaticamente, è il primo pensiero che mi si è presentato alla mente quando, riflettendo sul tema, constatavo che, se recepito dalla Tunisia, paese dove vivo e lavoro, un tale tema diventava, di fatto, privo di senso. Immerso nella realtà sociale e accademica tunisina, un simile interrogativo mi risultava, in effetti, improponibile e quasi surreale, un po' come se ci si volesse interrogare sulla diffusione degli sport invernali nelle dune del deserto di Douze. Ma constatare che in molte zone del mondo la diffusione e la ricezione della recente letteratura italiana è praticamente assente non riduce certo l'interesse per il tema, anzi ne aumenta forse le implicazioni. Mentre sarebbe ovviamente demenziale dedicare del tempo a riflettere sull'assenza di piste da sci in Tunisia, ragionare sui fattori che impediscono od ostacolano la diffusione della recente letteratura italiana può forse condurci, in effetti, ad affrontare delle questioni che assumono una pertinenza più generale $\mathrm{e}$ - oserei dire - globale. Questo è ancor più vero se il ragionamento si svolge da un paese come la Tunisia, dove l'ostacolo non può certo essere identificato nell'estraneità o nell'estrema distanza culturale. Per ovvie ragioni geografiche, oltre che storiche, tra i due paesi esistono profonde "contaminazioni"1 e la

1. Limitandoci alla storia recente, un fatto interessante da ricordare è che nel 1960, in seguito ad accordi di cooperazione, il governo italiano installò il primo ripetitore e contribuì a realizzare una rete di telediffusione terrestre. Da allora, il primo canale RAI è trasmesso in chiaro sulla quasi totalità del territorio nazionale tunisino e per alcuni decenni, prima del digitale e delle paraboliche, Rai1 è stato il canale televisivo più seguito, contribuendo così alla diffusione della lingua italiana e agendo sull'immaginario di intere generazioni. 
lingua italiana, studiata sia nei licei che in molte università, è, dopo il francese, la lingua straniera più parlata nel paese.

Nelle pagine che seguono, vorrei appunto provare ad analizzare le molteplici ragioni di quello che, ironizzando un po', si potrebbe chiamare un "incontro impossibile". Prima di considerare la situazione specifica della Tunisia, mi sembra però necessario inquadrare, anche se in modo sommario, il contesto generale in cui il termine letteratura assume oggi un senso, considerandola non solo in quanto creazione artistica, ma anche nella sua doppia natura, da un lato di sapere che viene trasmesso nelle scuole, e dall'altro di merce prodotta da un sistema industriale da cui dipendono, in gran parte, i processi di valorizzazione e di diffusione delle varie opere letterarie. Tralasciando il tema della crisi delle discipline letterarie e delle scienze umane in generale, su cui però tornerò in seguito riferendomi alla Tunisia, l'aspetto da cui mi pare necessario partire è la natura di merce della letteratura, che è poi il modo concreto con cui essa intesa materialmente come corpus di testi cartacei o meno - si presenta al pubblico. È a questo livello che negli ultimi trent'anni, grazie al connubio tra sistema industriale e media digitali, è avvenuto un vero sconvolgimento, causato da un'impressionante accelerazione produttiva che ha avuto come effetti la proliferazione dissipativa degli oggetti e l'emergere di una grande quantità di obsolescenza. Accelerazione del ritmo produttivo e novità permanente sono in realtà una tendenza generale del nostro mondo, e se tale tendenza si rivela distruttiva per l'ambiente lo è forse ancora di più quando investe la dimensione simbolica e culturale delle comunità umane. D'altra parte, come il filosofo Bernard Stiegler ha perfettamente mostrato, è proprio la cultura, nel senso lato del termine, a costituire oggi il cuore della politica industriale che ha, di fatto, totalmente assorbito la sfera dell'otium (termine con cui Stiegler designa il mondo dello spirito in generale) nella logica del negotium ${ }^{2}$, basata sul calcolo del profitto a breve termine. Per sollecitare il consumo, obiettivo primario del mercato globale, è infatti necessario catturare l'energia libidinale dei potenziali consumatori e questo non può avvenire se non agendo sulla sfera del simbolico e dell'immaginario. Il flusso ininterrotto di merci e di prodotti culturali conduce, secondo Stiegler, a una condizione di miseria simbolica in cui i simboli non vengono più "praticati", ma solo consumati. Una situazione che causa malessere e disorientamento, poiché vivendo in una tale condizione gli individui perdono il sentimento della loro singolarità, del mondo che li circonda e, con esso, la fiducia in

2. Tra i numerosi lavori di Bertrand Stiegler, mi riferisco in particolare a De la misère symbolique, 2 voll., Paris, Galilée, 2005. 
un avvenire da costruire. Stiegler non è certo il solo a sottolineare il ruolo centrale dell'industria culturale nell'attuale sistema produttivo e, a tal proposito, sono particolarmente pregnanti le considerazioni di Fredric Jameson che, in Postmodernismo o la logica culturale del capitalismo tardivo, osserva come la fusione della sfera culturale con quella economica non significhi l'umiliazione della cultura, ma al contrario la sua apoteosi, il suo trionfo, e questo nel senso che

la dissoluzione della sfera autonoma della cultura va immaginata piuttosto in termini di esplosione: un'immensa espansione della cultura nell'intero ambito sociale, al punto che si può dire che tutto nella nostra vita sociale - dal valore economico al potere statale fino alle pratiche e alla stessa struttura della psiche - sia diventato "culturale" in un senso originale mai prima teorizzato ${ }^{3}$.

Se ho fatto brevemente riferimento a questo genere di analisi (su cui peraltro, anche se partendo da prospettive diverse, si è attardato un gran numero di autori), è per evidenziare il fatto che la letteratura, in termini puramente quantitativi ed economici di libri prodotti e venduti, non è per nulla in crisi, ma è anzi perfettamente integrata nella logica produttiva del flusso continuo, il che poi significa, concretamente, il perenne rinnovo delle offerte e la riduzione dei tempi di permanenza dei libri a poche settimane. La conseguenza diretta di questo processo di fluidificazione, che è poi il dato forse più evidente della dinamica sociale che si è andata costruendo in questi ultimi decenni, è che nessun fenomeno o contenuto culturale riesce a costituirsi come vissuto collettivo e a iscriversi in una certa durata. Il ritmo del sistema produttivo inghiotte rapidamente $\mathrm{i}$ contenuti che esso stesso produce e di cui si alimenta, impedendo di fatto che questi si depositino e sedimentino nella memoria sociale. In ambito letterario, un aspetto particolarmente rilevante è che nel contesto attuale, la quantità di prodotti richiesta è talmente smisurata che si è allargata la base dei produttori riconosciuti, introducendo nel ciclo di produzione anche il cosiddetto contenuto generato dall'utente, ovvero i prodotti di chi, fino a ieri, componeva il pubblico, l'oggetto e il target della produzione. Il massiccio ricorso ai contenuti generati dagli utenti, che spesso significa il puro sfruttamento delle energie e del capitale cognitivo dei singoli individui da parte delle industrie, comporta poi un salto ben più radicale dell'azzeramento delle differenze tra cultura "alta" e cultura "bassa", di cui si dibatteva fino a pochi anni fa. Nel momento in cui ogni prodotto estetico viene riportato al grado zero del suo

3. Jameson, Fredric, Postmodernismo o la logica culturale del capitalismo tardivo, trad. it. M. Manganelli, Roma, Fazi, 2007, p. 192. 
valore di contenuto, non solo ogni gerarchia corrente viene smontata, ma si deve accettare anche l'impossibilità di istituirne altre. Ciò che ne segue non è una rielaborazione, anche radicale, del canone, ma il formarsi di un contesto in cui il canone non è istituibile. O meglio, di un contesto in cui la sola autorità che definisce il canone è il mercato e che, a differenza delle autorità precedenti, non lascia spazi a una sua critica o a una sua rimessa in discussione. Semplificando, potremmo dire che mentre "il canone accademico" pensava al valore in termini di durata, e per ciò stesso si prestava alla critica e alla revisione, il "canone mercantile", che identifica il valore nel calcolo matematico del profitto immediato, è sottratto a ogni tipo di critica.

La totale implosione del canone, avvenuta nel corso degli ultimi decenni, è in realtà dovuta a una serie complessa di dinamiche e di processi sociali che trascendono la dimensione strettamente economica e che sarebbe fuori luogo tentare di esporre qui in modo esaustivo, ammesso che questo sia possibile. Credo però che soffermarci a considerare alcuni di questi processi sia utile anche per poterci poi dedicare alle ragioni di quell'incontro impossibile, di cui si diceva all'inizio, tra letteratura italiana degli anni 2000 e realtà tunisina. Una tendenza particolarmente rilevante, e per certi versi paradossale, è data dal fatto che la narrazione, tradizionalmente considerata una componente essenziale dell'esperienza letteraria, è diventata una nozione centrale in ambiti anche assai lontani tra loro. In realtà viviamo avvolti in una narratività diffusa, che nella nostra epoca digitale contraddistingue aree importanti e normalmente ritenute extranarrative, come il marketing o la politica, e se la letteratura ha perso la sua posizione di prestigio nell'ambito del sapere, discipline quali la medicina o le scienze giuridiche si avvalgono sempre più di alcuni dei suoi concetti e dei suoi metodi d'analisi. Se questa narrazione espansa ha probabilmente contribuito a rendere più fluido il concetto di letteratura, a ibridarne i linguaggi e a farla assimilare a concetti più generici come immaginario, discorso o comunicazione, è però innegabile che il fattore scatenante siano stati i nuovi media e in particolare $\mathrm{i}$ social network. Su questo punto è interessante osservare come in un saggio intitolato Quanto vale e quanto dura un canone?, Cesare Segre abbia spiegato che nella nostra epoca di multimedialità le frontiere tradizionali tra i generi artistici cadono, perché i loro "elementi costitutivi si scambiano l'un l'altro in un continuo movimento magmatico". Da ciò, il critico ne deduce che "non essendo più alla testa delle cose letterarie autori o istituzioni culturali, questi movimenti saranno governati soltanto da un'imprenditoria anonima", una situazione che è in sé preoccupante, tenendo conto del fatto che "nessuno ha mai reclamato la proprietà collettiva dei mezzi di comunicazione, come si faceva una volta per la 
proprietà dei mezzi di produzione" ${ }^{\text {. }}$. A tutto questo va aggiunto che, nel contesto attuale, per sopravvivere alla competizione e non essere rapidamente dimenticati, scrittori e poeti devono costruire il proprio destino "fuori dal testo": network, gestione di pagine e account sui social incidono sulla popolarità di un autore quanto e più dei contenuti di ciò che effettivamente scrive, e questo fenomeno si accompagna all'indebolimento delle distinzioni fra forme letterarie e non, in quanto il concetto di testo letterario è diventato più fluido, e spesso si interseca con altri media e forme di discorso online. Non è un caso se autori come ad esempio Houellebecq in Francia, Roberto Saviano o Elena Ferrante in Italia, indipendentemente dalla qualità della loro scrittura, abbiano raggiunto una notevole notorietà grazie a fattori concomitanti ed extra-letterari. Rispetto alla narratività espansa e all'ibridazione dei linguaggi, mi pare inoltre che un elemento davvero saliente, cui il diffondersi dei social network ha dato luogo, sia la totale inversione del classico schema borghese che separava la sfera pubblica da quella privata: mentre da un lato il privato delle vite (pensiamo, tanto per fare un esempio, alle emozioni e ai pensieri di Madame Bovary che scandalizzavano la morale puritana della borghesia) si pubblicizza e si racconta nelle pagine di Facebook o di Twitter, dall'altro il pubblico si privatizza (anche nel senso concreto di interi settori dello Stato) e gli organi del potere diventano sempre più opachi e inintelligibili.

In questo elenco assai schematico dei fattori che hanno contribuito alla dissoluzione del canone, è però necessario considerare anche delle ragioni che hanno agito all'interno del campo letterario stesso. Un ruolo di rilievo in questo senso va certo riservato all'azione critica dei Cultural e Postcolonial Studies, che hanno giustamente contribuito a evidenziare la natura implicitamente eurocentrica dei criteri di valore con cui venivano giudicati gli oggetti della produzione artistica. È tuttavia possibile osservare che il loro iniziale impulso, per così dire sovversivo, si è ormai istituzionalizzato e ciò che spesso ne resta è la totale evacuazione del problema, ineludibile per ogni attività critica o interpretativa, del distinguere, del valutare e del giudicare.

Insieme al canone si è naturalmente dissolta la visibilità sociale della critica letteraria che, devalorizzata e ridotta ai margini dell'industria culturale, si rivela incapace, anche a causa dell'immensa quantità di merci letterarie che quotidianamente vengono immesse nel mercato, di offrire indicazioni o criteri interpretativi. È certo possibile, come del resto da più parti è stato rilevato, attribuire alla crisi della critica anche delle cause intrinseche e collegarla alla sua eccessiva

4. Segre, Cesare, Critica e critici, Torino, Einaudi, 2012, p.152. 
rarefazione e autoreferenzialità in metateorie - strutturalismo, formalismo, analisi semiotiche - che avevano l'ambizione di rendere interamente oggettivo e scientifico il metodo della lettura e dell'interpretazione letteraria. Possiamo però constatare che nonostante la relativa perdita della loro egemonia la critica letteraria non si è per nulla sollevata dalla propria crisi, la quale è probabilmente l'epifenomeno di un processo più vasto che non colpisce solo una disciplina, un metodo o un'istituzione (la critica letteraria nelle forme storiche in cui l'abbiamo conosciuta), ma l'esercizio stesso dello spirito critico tout-court. Quel che è inevitabile riconoscere è che l'autorevolezza della critica letteraria non è mai stata così bassa, inferiore persino a quella della critica gastronomica, dove almeno le tre forchette assegnate dalla guida Michelin offrono la garanzia di una qualche oggettività. Rendersi conto che un certo tipo di autorità tradizionale, quella dell'accademia e dei critici di professione con il relativo canone da essi stabilito, sta perdendo il proprio ruolo non è, in sé, un fatto necessariamente negativo o da rimpiangere con nostalgia. Il problema che vorrei sottolineare non riguarda però il "tipo" di canone o la forma specifica dell'istituzione deputata a costituirlo, ma la modalità con cui il canone, i criteri del giudizio, si formano in seno al tessuto sociale. Resta infatti aperta la questione, enorme, di sapere su quali basi sociali si formuleranno in futuro i giudizi estetici e se sia effettivamente auspicabile delegare questo ruolo al mercato, ovvero a un manipolo di addetti ai lavori e professionisti del marketing che avranno un profilo storicamente assai diverso da quello dei loro predecessori, dato che, come è facile prevedere, saranno formati in un sistema scolastico segnato da continue riforme e tagli alla ricerca e sottomessi al perenne ricatto di un mercato del lavoro fluido e precario.

Quanto esposto finora sulla situazione generale della letteratura e della critica è naturalmente valido anche per il contesto italiano, che rispetto ai processi di distribuzione e diffusione nel mercato mondiale deve inoltre fare i conti con una lingua nazionale accessibile a un pubblico numericamente assai inferiore a quello delle letterature anglofone o francofone. Evito di pronunciarmi sull'accusa di eccessivo provincialismo che, secondo alcuni osservatori, caratterizzerebbe la nostra narrativa nazionale contemporanea, osservando però che, anche se la riconoscessimo come valida, indicherebbe in ogni caso una particolare configurazione storica e non certo una peculiarità genetica, come del resto lo dimostrano fenomeni culturali della storia recente quali il cinema neorealista o autori come Calvino e Pasolini, tanto per citare degli esempi emblematici. Vorrei invece riferirmi brevemente a due autori che a mio avviso hanno recentemente individuato due aspetti effettivamente presenti in quello che è oggi il 
panorama letterario italiano. Due aspetti che, senza essere in contraddizione tra loro, hanno però l'effetto di autoannullarsi. Il primo è Raffaele Donnarumma che, dopo aver dichiarato l'avvenuto esaurimento della poetica postmoderna, rileva come sia oggi possibile constatare il ritorno, sebbene attraverso modalità rinnovate, a forme di realismo ${ }^{5}$. Il secondo autore è Alberto Asor Rosa che nel suo ultimo lavoro, Scrittori e Massa ${ }^{6}$, oltre ad ammettere che di fronte a una massa talmente grande di fenomeni letterari (recensisce circa 280-300 autori nati dopo il 1960 cui corrispondono circa 1000 titoli apparsi negli ultimi vent'anni), il lavoro del critico si rivela praticamente impossibile, osserva che se il disagio rappresenta un tema costante tra i giovani scrittori, questo disagio si presenta attraverso una sorta di "individualismo molecolare", il quale si concretizza in storie che non riescono mai a farsi portatrici di una Storia collettivamente intesa. La domanda che sorge è se, al di là del precariato, dell'estrema fluidificazione delle forme di vita e dell'appartenenza agli stessi dati anagrafici, esista per gli scrittori nati negli anni '80 una vera coesione generazionale, un evento simbolico o culturale capace di farli sentire figli di una storia comune, come per esempio era avvenuto per la generazione che aveva vissuto i movimenti del '68 e degli anni successivi. A giudizio di Asor Rosa, ciò che affiora dalle narrazioni delle ultime generazioni di scrittori è il "locale", di cui però non si avverte mai il "nazionale" e da cui molto probabilmente non si configurerà, negli anni a venire, alcun "classico".

Indipendentemente dalla problematicità del termine "classico", è forse lecito chiedersi se esistono oggi le condizioni per l'emergere dell'opera "esemplare", se sia ancora possibile parlare oggi di "letterature nazionali" e se, o in che modo, questa situazione costituisca un limite e non, al contrario, l'opportunità per superare alcuni schemi categoriali ormai inadeguati ${ }^{7}$.

5. Il riferimento a Donnarumma è dovuto principalmente all'interessante dibattito da lui animato nel 2008 sul numero 57 della rivista Allegoria intitolato, appunto, Ritorno alla realtà? Narrativa e cinema alla fine del postmoderno. La rivista è consultabile in rete all'indirizzo : http://www.allegoriaonline.it/index.php/i-numeri-precedenti/allegoria-n57. Sebbene il consenso non sia unanime, gli autori che indicano un ritorno al realismo dopo la fase postmoderna sono comunque numerosi. Si veda, per esempio, FERRARIS, Maurizio, Manifesto del nuovo realismo, Roma, Laterza, 2012.

6. Asor Rosa, Alberto, Scrittori e massa, Torino, Einaudi, 2015. Il volume, in realtà, riproduce il noto saggio del 1965, Scrittori e popolo, e la parte dedicata al contemporaneo, cioè Scrittori e massa, occupa solo le ultime sessanta pagine, forse anche a dimostrazione di quel disorientamento che emerge dalle sue pagine.

7. Tentando di rispondere a questo genere d'interrogativi, alcuni autori hanno proposto di far rivivere l'idea goethiana di una letteratura mondiale (vedi, per esempio, Casanova, Pascale, La République mondiale des lettres, Paris, Éditions du Seuil, 1999; 
Il quadro fin qui tratteggiato è certo superficiale e descrive una serie di dinamiche sociali complesse che queste pagine non possono certo circoscrivere, ma mi pare sia sufficientemente realistico per poterne dedurre alcune considerazioni generali. Possiamo innanzitutto costatare che se da un lato la produzione di testi letterari in questi ultimi anni è vertiginosamente aumentata, quella che invece è venuta meno è una "società letteraria", nel senso che quest'espressione poteva avere fino a qualche decennio fa e indipendentemente dal valore negativo o positivo che le si può attribuire. Senza stabilire dei semplici nessi causali, possiamo inoltre osservare che insieme alla "società letteraria", da intendersi genericamente come istanza mediatrice tra produzione e ricezione, si è dissolto quell'apparato normativo che veniva generalmente sintetizzato nella nozione di canone, e che intellettuali e scrittori navigano a vista, depositando ciascuno il proprio contributo in quell'immenso magma della produzione culturale, artistica e letteraria che, almeno apparentemente, si fa da sé. Tutto questo si accompagna, in modo piuttosto naturale, alla devalorizzazione dei saperi letterari, delle istanze scolastiche o universitarie che si occupano della loro trasmissione e della critica, di cui la società sembra non avere più un gran bisogno dato che l'industria ne ha agevolmente assorbito il ruolo.

Se ho dedicato una buona parte di questo saggio a considerazioni generali sullo statuto attuale della letteratura e dei saperi letterari, è perché ciò che chiamiamo globalizzazione significa anche, in gran parte, globalizzazione dell'industria culturale e progressiva omogeneizzazione dei sistemi d'insegnamento. Nonostante le specificità locali, si tratta di un processo che riguarda il mondo intero e che, nel caso della Tunisia, si presenta con caratteri ancora più accentuati. È in gran parte da questo dato di fatto che è necessario partire per comprendere quali ostacoli impediscano alla narrativa italiana degli anni 2000 di attraversare quello strettissimo lembo di mare che separa l'Italia dalla Tunisia, due paesi uniti da profondi legami storici e culturali. Bisogna poi ricordare che a causa della divisione internazionale del lavoro, la devalorizzazione e la progressiva riduzione di fondi per le discipline umanistiche sono tanto maggiori quanto più ci s'inoltra nei paesi del sud del mondo. La scarsità di risorse intensifica la tendenza - planetaria - ad allineare i programmi d'insegnamento alle esigenze delle imprese e del mondo del lavoro, riducendo al massimo gli inve-

MoretTi, Franco, Opere mondo, Torino, Einaudi, 2003). La proposta ha però suscitato numerose critiche, soprattutto nell'ambito degli studi postcoloniali. Sul tema, cfr. SpIvak, Gayatri, Morte di una disciplina, Roma, Meltemi, 2003, e il volume ben documentato di Benvenuti, Giuliana, Ceserani, Remo, La letteratura nell'età globale, Bologna, Il Mulino, 2012. 
stimenti nelle discipline giudicate improduttive, tra le quali gli studi letterari occupano l'ultimo posto. Nel caso della Tunisia, va poi aggiunto che la scelta degli studi universitari non è libera, ma dipende dalla valutazione ottenuta alla fine degli studi superiori: voti alti significano poter accedere a discipline scientifiche o economiche, voti mediocri permettono l'accesso unicamente a discipline umanistiche. Il gradino più basso è costituito proprio dalle facoltà letterarie e sono davvero pochissimi gli studenti che scelgono liberamente di seguire tale tipo di studi, mentre per la stragrande maggioranza si tratta di una scelta obbligata intrapresa non solo senza avere un particolare interesse, ma con la consapevolezza della scarsa considerazione sociale per questi indirizzi disciplinari e dell'altissima percentuale di disoccupati che producono. Per pressioni e promesse di aiuti e finanziamenti da parte della Banca Mondiale e del FMI, dal 2006 la Tunisia ha adottato la riforma del sistema universitario denominata LMD e promossa dal ciclo d'incontri tra ministri europei dell'istruzione cominciato a Bologna nel 1999. Se in Europa gli effetti negativi di tale processo di riforma sono ormai riconosciuti in modo pressoché unanime, in Tunisia la riforma ha prodotto conseguenze ancora più nefaste perché, oltre ad aver drasticamente ridotto i piani di studio, ha dato luogo a un proliferare di master dai titoli spesso fantasiosi, senza che questi siano accompagnati da un'effettiva formazione dei docenti o da materiali didattici adeguati. I corsi di letteratura impartiti durante il triennio della licence sono ridotti al minimo e distribuiti in pochissime ore d'insegnamento durante un anno accademico diviso in due semestri che si riducono, di fatto, a due mesi reali di lezioni. In parte per l'obbligo di rispettare i programmi ministeriali e in parte per le difficoltà oggettive ad aggiornarsi, a reperire i testi e a orientarsi nella massa della produzione letteraria contemporanea, cui manca, per le ragioni già esposte, un coerente e corposo apparato critico, i docenti tendono a impostare i loro corsi su un sapere consolidato e a proporre o delle sintesi della storia letteraria o selezioni tratte da autori appartenenti alla tradizione classica. Uno sguardo alle tesine di master o ai lavori di dottorato è sufficiente per rendersi conto che tutte le ricerche hanno come limite temporale autori dell'immediato dopoguerra e che tutto ciò che è avvenuto dopo gli anni '80 è avvolto da una fittissima nebbia. Negli studenti, generalmente pochissimo abituati alla lettura, si consolida in questo modo un'idea della letteratura come monumento e patrimonio, cioè un sapere legato a un passato che ha scarse relazioni con il loro vissuto e il loro mondo. La narrativa ultra-contemporanea è dunque perlopiù assente dai programmi di studio. Inoltre, nella percezione generale, data l'assenza di criteri di selezione o d'orientamento, la massa di titoli e romanzi pubblicata da giovani autori è indistintamente 
livellata allo statuto di merci che l'industria culturale immette costantemente nel mercato e destinate ad essere consumate o meno secondo le propensioni individuali che ci fanno emettere un giudizio sensibile basato sull'alternativa "mi piace/non mi piace", ma senza la possibilità di passare dal sensibile all'intellegibile, dal privato al pubblico, dall'idiosincratico al comunicabile.

Nel caso specifico della Tunisia, bisogna inoltre tener conto del fatto che le traduzioni in arabo sono praticamente inesistenti e quindi l'accesso alla letteratura italiana è possibile o in lingua originale o in traduzioni francesi, ossia in modo sempre mediato da una lingua straniera, e questo crea inevitabilmente ulteriori difficoltà alla penetrazione della recente narrativa italiana. Nelle librerie esistenti a Tunisi, sono effettivamente rari gli autori italiani disponibili, tra l'altro con un costo dei libri che risulta generalmente proibitivo per il pubblico tunisino. Se poi osserviamo le attività delle due istituzioni italiane presenti in Tunisia e deputate alla diffusione della cultura italiana, l'Istituto Italiano di Cultura e la Società Dante Alighieri, l'impressione è egualmente scoraggiante. Entrambe le istituzioni organizzano corsi di lingua e dispongono di una biblioteca, ma se quella della Dante ha un'impronta prevalentemente storica e centrata sulla memoria della presenza italiana in Tunisia (su cui peraltro si sta compiendo un grande lavoro di ricostruzione), quella dell'Istituto, di dimensioni assai ridotte, è ben poco aggiornata e la letteratura degli anni 2000 è perlopiù assente. Nel complesso possiamo quindi dire che nonostante l'iniziativa e la passione dei singoli docenti, le possibilità di fare ricerca e di recuperare materiale didattico sono estremamente difficili; e di fronte a tali difficoltà si finisce spesso per soccombere alla facile soluzione di ripercorrere strade già ampiamente battute cui viene apportato pochissimo valore aggiunto.

Lo scenario fin qui esposto non è certo positivo, ma vorrei concludere tentando di dissipare l'eventuale impressione che esso sia il frutto di una visione catastrofica o apocalittica del mondo. Se da un lato sono convinto che esistano innegabili e fondate ragioni per provare un senso di disagio e di disorientamento - e l'impiego generalizzato del termine "crisi" per caratterizzare una molteplicità di dimensioni del vivere in comune sta lì a dimostrarlo - dall'altro penso che sarà proprio dalla crisi, dalla coscienza di un disorientamento, soprattutto se si tratta di un sentimento condiviso collettivamente, che potranno sorgere nuovi criteri, nuove tracce, capaci di ridare un senso e una direzione al mondo, e capaci di far fronte, capovolgendola, alla tendenza entropica che sembra oggi attraversarlo. Anche se l'egemone e potentissima ideologia del libero mercato sembra aver ormai fagocitato l'intero pianeta, penso che nessuna ideologia possa riuscire a penetrare e a modellare, senza resti, la moltitudine delle 
coscienze. Esiste sempre un eccesso, un supplemento, cioè delle forme variegate di resistenza che da potenza si trasformano in forza quando il sentire delle coscienze individuali si coagula in un sentire collettivo. Nonostante la fine dichiarata delle grandi narrazioni, sono convinto che anche oggi, e forse più di prima, la dimensione narrativa sia la forma principale attraverso cui molteplici volontà particolari possono fondersi in una volontà più generale, e condivido pienamente l'idea del collettivo Wu Ming, secondo cui non può esserci alcuna lotta contro lo stato di cose presenti se non si è ispirati da una qualche narrazione. Credo quindi che concentrare l'attenzione sulla dimensione narrativa, da intendersi non solo come sapere letterario consacrato, ma anche come attività pratico/teorica che permette la costituzione di singolarità individuali e collettive, sia un compito davvero essenziale per immaginare e costruire le nostre società a venire.

Mario SeI

Université de la Manouba 
